\title{
Correction to: Investigation of Functional Analysis Methodology in Adult Service Programs to Develop Efficient and Effective Treatment Approaches
}

\author{
Cynthia M. Anderson ${ }^{1} \cdot$ Sarah A. Weddle ${ }^{1}$ (D) Margaret L. Walsh ${ }^{1} \cdot$ Jaclyn Guglielmo ${ }^{1}$ \\ Published online: 6 August 2019 \\ (C) Springer Nature Switzerland AG 2019
}

\section{Correction to: Advances in Neurodevelopmental Disorders} https://doi.org/10.1007/s41252-019-00118-w

The authors would like to correct three errors, none of which change the conclusions or interpretations presented. First, the text within the condition column label on Table 1 does not align with the corresponding description of the condition. The condition labels should be aligned with the description of the functional analysis conditions tested for each participant. Second, the symbol key for Fig. 1 was missing the labels which identify the condition being tested in the standard functional analysis. For both we neglected to detect the omissions and formatting errors that occurred when the final submission was converted to a proof. Third, Carlie's problem behavior for session 1 occurred at 2.4 responses per minute, and not 2.7 responses per minute as Fig. 1 depicted in the original article. This was an unintentional error during data entry.

The original article has been corrected.

The online version of the original article can be found at https://doi.org/ 10.1007/s41252-019-00118-w

Cynthia M. Anderson

canderson@mayinstitute.org

1 May Institute, Randolph, MA, USA 
Table 1 Description of standard functional analysis (FA) and IISCA conditions for Carlie, Parker, and Whitney

\begin{tabular}{|c|c|c|c|c|}
\hline \multirow[t]{2}{*}{ Participant } & \multicolumn{2}{|c|}{ Standard FA } & \multicolumn{2}{|l|}{ IISCA } \\
\hline & Condition & Description & Condition & Description \\
\hline \multirow[t]{5}{*}{ Carlie } & Escape & $\begin{array}{l}\text { EO: sorting colored socks } \\
\text { C: } 20 \text {-s break }\end{array}$ & \multirow[t]{4}{*}{$\begin{array}{l}\text { Escape to attention } \\
+ \text { tangible test }\end{array}$} & $\begin{array}{l}\text { EO: removal of tablet, sorting colored socks, diverted } \\
\text { attention }\end{array}$ \\
\hline & Tangible & $\begin{array}{l}\text { EO: tablet removed } \\
\text { C: } 20 \text {-s access to tablet }\end{array}$ & & $\begin{array}{l}\text { C: } 30 \text {-s break from task, statement of concern, access to } \\
\text { tablet for } 30 \text {-s, redirections and statements of }\end{array}$ \\
\hline & \multirow[t]{2}{*}{ Attention } & EO: attention diverted & & \\
\hline & & $\begin{array}{l}\text { C: } 20 \text {-s redirections and statements of } \\
\text { concern }\end{array}$ & & \\
\hline & Control & $\begin{array}{l}\text { Continuous attention and tablet, no } \\
\text { demands }\end{array}$ & Control & Continuous attention and tablet, no demands \\
\hline \multirow[t]{5}{*}{ Parker } & \multirow[t]{2}{*}{ Escape } & $\begin{array}{l}\text { EO: fine motor demand with modeling } \\
\text { clay (roll into forms) }\end{array}$ & \multirow[t]{4}{*}{$\begin{array}{l}\text { Escape to } \\
\text { attention test }\end{array}$} & $\begin{array}{l}\text { EO: fine motor demand with modeling clay/stuffing enve- } \\
\text { lopes \& diverted attention }\end{array}$ \\
\hline & & C: 30 -s break & & C: $30-s$ break with redirection and statement of concern \\
\hline & \multirow[t]{2}{*}{ Attention } & EO: diverted attention & & \\
\hline & & $\begin{array}{l}\text { C: } 30 \text {-s redirection and statement of con- } \\
\text { cern }\end{array}$ & & \\
\hline & Control & $\begin{array}{l}\text { Continuous attention, preferred activity } \\
\text { (shredding papers), no demands }\end{array}$ & Control & $\begin{array}{l}\text { Continuous attention, preferred activity (shredding papers), } \\
\text { no demands }\end{array}$ \\
\hline \multirow[t]{5}{*}{ Whitney } & \multirow[t]{2}{*}{ Escape } & $\begin{array}{l}\text { EO: fine motor task (inserting dominoes } \\
\text { into a container with opening) }\end{array}$ & \multirow[t]{4}{*}{$\begin{array}{l}\text { Escape to } \\
\text { attention test }\end{array}$} & $\begin{array}{l}\text { EO: fine motor task (inserting dominoes into container with } \\
\text { opening) \& diverted attention }\end{array}$ \\
\hline & & C: 20 -s break & & C: 30 -s break from task, redirection and statement of concern \\
\hline & \multirow[t]{2}{*}{ Attention } & EO: diverted Attention & & \\
\hline & & $\begin{array}{l}\text { C: } 20 \text {-s redirections or statements of con- } \\
\text { cern }\end{array}$ & & \\
\hline & Control & $\begin{array}{l}\text { Continuous attention, preferred tangible } \\
\text { (bean bag chair), no demands }\end{array}$ & Control & $\begin{array}{l}\text { Continuous attention, preferred tangible (bean bag chair), no } \\
\text { demands }\end{array}$ \\
\hline
\end{tabular}

$E O$ establishing operation for problem behavior. $\mathrm{C}$ consequence provided contingent on problem behavior. 
Fig. 1 Problem behavior per minute for standard FA and

IISCA for Carlie, Parker, and Whitney
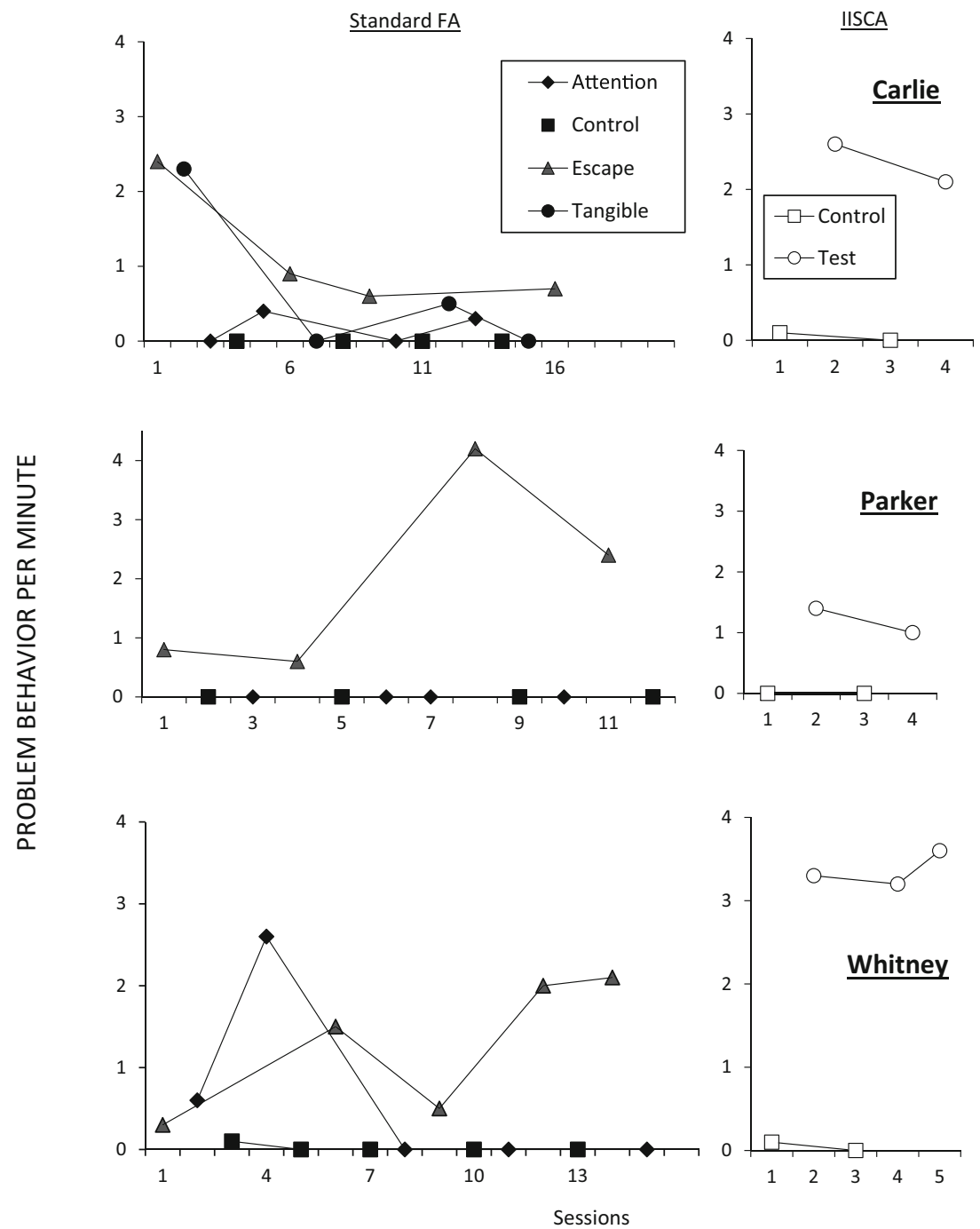

Publisher's Note Springer Nature remains neutral with regard to jurisdictional claims in published maps and institutional affiliations. 